Life Sciences: Oral 3

\title{
Morphologic characterization of Mycobacterium tuberculosis circulating strains in a Lisbon hospital
}

\author{
C. Silva ${ }^{1}$, E. Alverca ${ }^{2}$, A.P. Matos ${ }^{3}$, P.A. Carvalho ${ }^{4}$,I. Portugal ${ }^{1}$, L. Jordao ${ }^{5}$ \\ ${ }^{1}$ Centro de Patogénese Molecular, URIA, Faculdade de Farmácia da Universidade de Lisboa, Av. Prof. Gama \\ Pinto, 1649-003 Lisboa, Portugal; \\ ${ }^{2}$ Departamento de Saúde Ambiental, Instituto Nacional de Saúde Dr Ricardo Jorge (INSA), Av. Padre Cruz, \\ 1649-016 Lisboa, Portugal; \\ ${ }^{3}$ Serviço de Anantomia Patológica, Centro Hospitalar de Lisboa Central, Hospital Curry Cabral, R. da \\ Beneficência 8, 1069-166 Lisboa, Portugal; \\ ${ }^{4}$ ICEMS, Departamento de Bioengenharia, Instituto Superior Técnico, Universidade Técnica de Lisboa, Av. \\ Rovisco Pais, 1049-001 Lisboa, Portugal \\ ${ }^{5}$ Departamento de Doenças Infeciosas, INSA, Av. Padre Cruz, 1649-016 Lisboa, Portugal; \\ email: maria.jordao@insa.min-saude.pt
}

Tuberculosis (TB) is one of the killers accounting for 3.1 million deaths per year. This disease caused by $M$. tuberculosis $(\mathrm{Mtb})$ was considered curable and preventable after the advent of antibiotics and the CalmetteGuérin bacillus vaccine. Nevertheless, during the last decades of the $20^{\text {th }}$ century TB made a deadly come back triggered mainly by the emergence of acquired immunodeficiency syndrome (AIDS). More recently, the emergence of multidrug resistant (MDR) and extensively drug resistant (XDR) Mtb strains, uncovered the most freighting face of this disease an incurable infection with the currently available therapeutic tools [1]. Although, Portugal is considered a TB low incidence setting, annually are reported MDR and even XDR TB cases. The majority of these cases occur in the Lisbon area and the strains involved belong to a well characterized genotypic family discovered in the 1990's know as Lisboa family [2].

In the present work we studied a group of Mtb strains collected in a Lisbon hospital during two years (20082009). The morphology of colonies grown on Lowenstein-Jensen (LJ) slants was studied by different electron microscopy (EM) techniques. The aim of the study was the establishment of a link between mycobacteria drug susceptibility and structure. A total of $283 \mathrm{Mtb}$ strains were collected during the period of the study. Among the isolates collected during 2008, 12\% were resistant to at least one first line anti-bacillar drug, $5 \%$ were MDR and 5.2\% XDR. During 2009, although the number of resistant and MDR strains raised to 19\% and $5.6 \%$, respectively, the percentage of XDR strains dropped to $2.8 \%$.

In the first part of the study approximately 20 mycobacteria strains, with different drug susceptibility profiles, were grown on LJ and their morphology was compared. Although all the mycobacteria strains originate rough colonies their size differ with the drug susceptibility profile. The pan-susceptible strains generate larger colonies than drug resistant strains as shown in figure 1. Next these colonies were studied by scanning electron microscopy (SEM). The results obtained show that mycobacteria surface are distinct in susceptible and drug resistant strains as shown in figure 2.A-D. While drug susceptible mycobacteria have a homogenous surface (Fig. 1A), drug resistant bacteria present a heterogeneous surface (Fig. 2B) with small protrusions (Fig. 2C).

In order to evaluate the existence of differences in the ultrastructure of circulating $M t b$ strains we started a study using transmission electron microscopy (TEM). For this approach were selected only two isolates (pansusceptible: R188/09 and XDR: HPV108/09) which were chemically fixed, post-fixed with osmium tetroxide and further processed for epon embeeding. The results obtained (Table 1) show that mycobacteria cell width 
$(\cong 350 \mathrm{~nm})$ is similar for both bacteria. Nevertheless, their cell length and cell envelope width are significantly different. The XDR strain is shorter $(p=0.009)$ and has a ticker cell envelope $(p=0.004)$ than the pansusceptible strain. These results are in good agreement with those published in the literature $[3,4]$.

Altogether our data clearly shows the existence of a link between mycobacteria ultrastructure and drug susceptibility. In order to better evaluate these differences a larger number of isolates must be studied. The use of other EM techniques, such as CEMOVIS, will avoid the formation of undesirable artefacts (e.g. mesossome) produced by dehydration and room temperature sectioning allowing a better characterization of mycobacteria ultrastructure [5].

The authors aknowledge the funding by Fundação para a Ciência e Tecnologia (SFRH/BD/73579/2010 and C2008-C2008_P2).

1. WHO 2011/ 2012 Tuberculosis Global Facts

2. Portugal I. Int J Tuberc Lung Dis 1999;3:207.

3. Takade A. Microb Immunol 2003; 47: 265.

4. Velayati AA. Chemotherapy 2009; 55:303.

5. Bleck CKE. J of Microscopy 2010; 237: 23.

Fig. 1 - Mycobacterium tuberculosis colonies grown on Lowenstein-Jensen slants. The colonies shown are representative of those obtained during the study. R188/09 is a pansusceptible Mtb strain and HPV108/09 is a XDR Mtb strain.
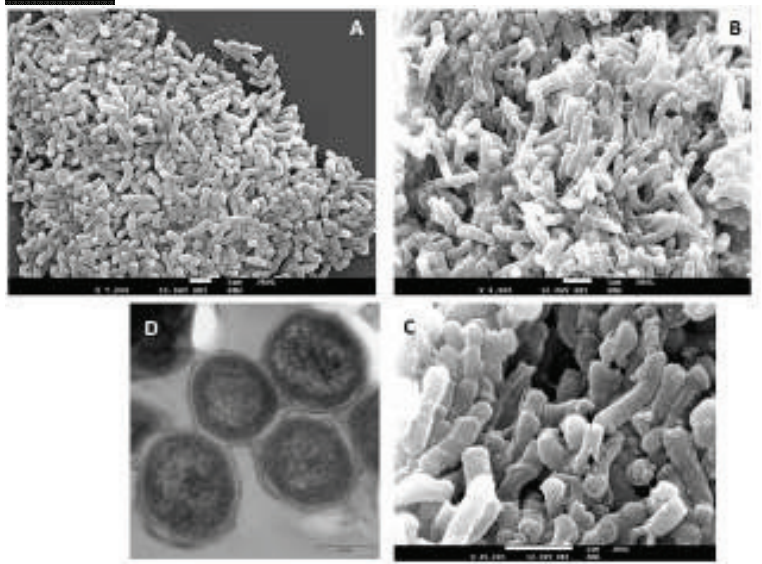

Fig. 2 - SEM and TEM images of Mycobacterium tuberculosis strains. SEM analysis of pansusceptible R108/09 (A) and XDR HPV188/09 (B, C) $M t b$ strains. The black arrows highlight the protrusions observed in the surface of HPV188/09 (C). TEM analysis was also performed for both isolates. A representative image is shown for HPV188/09 (D). For SEM Bar $=1 \mu \mathrm{m}$ and for TEM $\mathrm{Bar}=0.2 \mu \mathrm{m}$.

\begin{tabular}{lcccccc}
\hline \multirow{2}{*}{$\begin{array}{c}M t b \\
\text { strain }\end{array}$} & \multicolumn{2}{c}{$\begin{array}{c}\text { Cell width } \\
(\mathrm{nm})\end{array}$} & \multicolumn{2}{c}{$\begin{array}{c}\text { Cell length } \\
(\mathrm{nm})\end{array}$} & \multicolumn{2}{c}{$\begin{array}{c}\text { Cell envelope } \\
(\mathrm{nm})\end{array}$} \\
\cline { 2 - 7 } & average & SD & average & SD & average & SD \\
\hline R 188/09 & 350,6 & 37,3 & 919,6 & 12,9 & 15,3 & 2,58 \\
HPV108/09 & 335,3 & 41,9 & 855,7 & 22,9 & 21,8 & 1,39 \\
\hline
\end{tabular}

Table 1. Measurement of mycobacteria cell envelope, cell width and length. 